\title{
Achieving patient priorities: an alternative to patient-reported outcome measures (PROMs) for promoting patient-centred care
}

\author{
Aanand D Naik (D) , ${ }^{1,2}$ Angela Catic ${ }^{1,2}$
}

'Department of Medicine, Baylor College of Medicine, Houston, Texas, USA

${ }^{2}$ Houston Center for Innovations in Quality, Effectiveness, and Safety (IQuESt), Michael E. DeBakey VA Medical Center, Houston, Texas, USA

\section{Correspondence to}

Dr Aanand D Naik, Department of Medicine, Baylor College of Medicine, Houston, TX 77030, USA; anaik@bcm.edu

Accepted 12 October 2020 Published Online First 28 October 2020

\section{Sinked}

- http://dx.doi.org/10.1136/ bmjqs-2019-010742

\section{Check for updates}

(c) Author(s) (or their employer(s)) 2021. No commercial re-use. See rights and permissions. Published by BMJ.

To cite: Naik $A D$, Catic $A$. BMJ Qual Saf 2021;30:92-95.
For the past two decades, patientcentredness has served as one of six acknowledged dimensions of healthcare quality. ${ }^{1}$ Initially, healthcare institutions described patient centredness superficially-clean waiting rooms, hotel-like bed and board, access to innovative medical technology-and measured it with crude satisfaction scales. The concept of patient-centred care evolved into a model attuned to the patient experience of care, defined by the interactions between patients and providers and the care environment. ${ }^{2}$ This patient experience model of patient-centred care has deep normative roots around principles of the patient as the locus of control and a demand for individualisation and customisation of care based on the patient rather than clinician. ${ }^{3}$ Empirically, patient experience is associated with health outcomes when defined and measured in a timely manner as a specific care experience or interaction between a patient and a healthcare provider. ${ }^{4}$ The importance of honouring the patient experience is now a widely appreciated construct and a common measure of healthcare quality with a deep evidence base. ${ }^{5}$ The Hospital Consumer Assessment of Healthcare Providers and Systems, Consumer Assessment of Health Providers and Systems Survey and Press Ganey patient satisfaction measures are ubiquitous measures of quality defining patient experiences of care.

\section{MOVING BEYOND PATIENT EXPERIENCE MEASURES}

The effort to transform healthcare systems from clinician to patient centred is not complete. Honouring, measuring and ameliorating patients' experiences of care is necessary but not sufficient and represents only the first stop on the journey to patient-centred care. ${ }^{6}$ The second stop is one that nests the locus of control with patients and caregivers. Patients' control over healthcare decisions is useful only when transparency exists in all aspects of care: evidence, costs, processes, outcomes and errors. ${ }^{3}$ Unfortunately, claims that patients should have control and transparent understanding of all aspects of care have largely been ignored due to institutional inertia, lack of financial incentives and the primacy of professionals. In essence, there are few incentives to change this orientation, and clinicians too often perceive confrontation and frustration rather than partnership. ${ }^{7}$

The primacy of physician professionalism stems from professional control over scientific knowledge and nurse professionalism from control over the practice environment, both bolstered by years of training and experience. This professional model held for nearly a century when acute illnesses were the primary reason people sought medical care with the assumption that treatments were focused on cure (return to health) and/or alleviation of symptoms (removal of the disease). ${ }^{8}$ In contrast, healthcare in the 21st century primarily focuses on managing chronic diseases for which there are few cures. In the context of multiple chronic conditions (multimorbidity), the desired outcomes of healthcare are no longer obvious because they extend beyond the goals of curing diseases or prolonging life. Multimorbidity also produces trade-offs among treatments, conditions and possible outcomes. For patients with multimorbidity, 
evidenced-based treatments are often lacking and, when present, there may be conflicts or incongruences across conditions. ${ }^{10}$ Effective management of chronic conditions requires active, ongoing participation by patients and caregivers outside of healthcare settings. The intensity of this management can be burdensome, further impacting patient experiences and even outcomes. ${ }^{11}$ Healthcare professionals now increasingly understand the need to share the burden of treatment decisions with their multimorbid patients.

\section{PATIENT CENTREDNESS AS HEALTHCARE THAT ACHIEVES PATIENT PRIORITIES}

The next stop on the journey to patient-centred care is the establishment of collaborative partnerships between healthcare professionals and patients. ${ }^{6}$ Productive partnerships require a medium for shared understanding that does not default to professional expertise and clinical practice guidelines. We have asserted that patient priorities are the necessary medium for focusing collaboration, discussions and healthcare decisions, especially in the context of complex, chronic illnesses. ${ }^{10}$ We precisely define patient priorities as the combination of the specific and realistic outcomes and activities (health outcome goals) that individuals want based on what matters most to them and the healthcare activities, including medications, self-care, tests and visits that they are willing and able to perform (healthcare preferences) to achieve their outcome goals. ${ }^{12}$ Evidence and professional judgement still guide which treatments are relevant, but clinicians should partner with their patients to select and adjust care based on a health goal as opposed to individual disease states. ${ }^{13}$ Pragmatic studies demonstrate that this patient priorities approach to care reduces polypharmacy and patient-reported treatment burden while increasing care that aligns with patient goals. ${ }^{14} 15$ Patients and clinicians describe this process as practical and beneficial. ${ }^{16}$

\section{MEASURING GOAL ATTAINMENT AS A PATIENT- CENTRED CARE QUALITY MEASURE}

To promote and disseminate patient priorities-aligned care, novel quality measures are necessary. These quality metrics would evaluate the process for collaboratively identifying patient goals and care preferences and the degree to which patient goals are attained. In the current issue of BMJ Quality and Safety, Giovannetti et $a l^{17}$ describe the results of an innovative study that evaluated the feasibility of two different approaches to developing quality measures of goals-based care. The study assessed the implementation of these measures into diverse clinical settings and the subsequent interpretability and usefulness of the measures based on the data generated from either approach.

As Giovannetti and colleagues describe, the key gap in evaluating goals-based care is the presence of measures for setting and documenting goals as well as tracking goal progress and attainment. ${ }^{17}$ In routine care, patient goals and care preferences are infrequently and haphazardly written and communicated, often conflicting, and typically focus on end-of-life care or chronic disease biomarkers. ${ }^{18-21}$ To address these gaps, the authors adapted goal attainment scaling, a reliable and valid approach for measuring goal setting and goal attainment in research studies. ${ }^{22}{ }^{23}$ The authors asked patients and clinicians to jointly set a goal and define a set of possible outcomes along a five-point scale. They later discussed and then individually rated the degree of goal attainment. The other approach evaluated by Giovannetti and colleagues ${ }^{17}$ is the use of patient-reported outcome measures (PROMs), which are often used to measure specific domains (eg, mood, functioning, symptoms and so on) of health-related quality of life. ${ }^{24-26}$ In their study, Giovannetti et $a l^{17}$ asked patients and clinicians to jointly set a goal and then select a PROM that best matches that goal. At follow-up, the patient completed the same PROM to assess change over time. Patients and clinicians were given a dozen PROMs from which to select.

The study design and results of the study by Giovannetti et al are both novel and provocative. The authors found that clinicians were more likely to implement goal attainment scaling, noted to be practical to implement, compared with the PROM approach. Furthermore, clinicians found goal attainment scaling more useful for determining which services and supports to recommend and for helping patients achieve their goals. Contrary to common assumptions, the authors found that clinicians and patients set goals collaboratively and focused on patient-centred outcomes rather than disease processes or biomarkers. These findings suggest that implementation of a goals-based approach in routine care is feasible and demonstrate promise for fostering the shift from disease to patient-centred care.

The lack of appeal for the PROM approach is surprising given their broad acceptance as quality measures. ${ }^{27}$ PROMs are effective tools for measuring particular behaviours, activities or symptoms that are either specific to a disease, such as diabetes, ${ }^{28}$ or reflect overall health-related quality of life. ${ }^{29}$ As quality metrics, PROMs provide patient-centred measures that can be applied across a population of patients, such as the Patient Health Questionnaire for measuring depression symptoms. However, patients and clinicians seem to prefer goals-based approaches, such as goal attainment scaling ${ }^{30}$ and patient priorities care ${ }^{10}$ because they better reflect the goals of specific individuals within the context of their own lives. We have shown that when older patients set goals that are specific to their individual lives, they typically fall into one of four health-related values categories: (1) social and spiritual connections, (2) functioning and independence, (3) life enjoyment and pleasurable activities and (4) balancing quality and quantity of life (managing health). ${ }^{3132}$ We have trained clinicians 
to identify specific and realistic goals based on what matters most to patients by initiating conversations around the four health values categories. ${ }^{12}$ These conversations can be efficiently incorporated in clinic visits and during telehealth encounters. In another clinical trial, we demonstrated that a patient goalsbased approach can significantly improve scores on a validated depression-specific PROM compared with routine guidelines-based care. ${ }^{33}$ These findings suggest that individualised approaches to goal attainment can be coupled with PROMs to provide a balanced (individualised goals along with population-level measures) approach to quality measurement of patient-centred care.

\section{FINANCIAL INCENTIVES TO PROMOTE PATIENT- CENTRED CARE}

To facilitate dissemination of patient priorities aligned care, health insurers should support targeted financial incentives to facilitate widespread adoption into routine care. First, time-based reimbursement for clinical encounters with patients is vital. Medicare's care management billing codes for annual wellness, advanced care planning and chronic care management are also potential options. Establishment of novel value-based care management codes that are specific to priorities setting and measuring goal progress and attainment would be key drivers of this effort. Furthermore, these codes should support involvement of a range of health professionals. Training opportunities supported by continuing education credits would further promote patient priorities care. Common concerns about quality measures focused on goal attainment include the setting of unrealistic or inappropriate goals, playing the system with easily attained goals and the nuances of patient-caregiver-clinician goal alignment. These are all practical challenges to achieving a mature goals-aligned care process. However, at this early stage of development, Medicare should promote all efforts to implement value-based care management codes even if they are used primarily for financial incentives. Any impetus that encourages goal-based conversations and goal setting among patients, caregivers and clinicians will promote the necessary paradigm shift from guidelines-based care to goals-based care even if it tolerates some gaming of incentives. The promise of patient values and goals as the driver of patient-centred care is now two decades in development. ${ }^{1}$ Pragmatic, empirically supported processes for identifying patient goals and preferences during routine care and aligning treatment decisions to achieve these patient priorities are a welcome addition to the literature. Medicare and health insurers must now respond with incentives and quality measures that promote this mature vision of patient-centred care.

Funding ADN receives support as a co-investigator on Patient Priorities Care from the John A. Hartford Foundation (PI:
Mary Tinetti, Yale University): www.patientprioritiescare.org. $\mathrm{ADN}$ and AC received support for dissemination of Patient Priorities Care from the Department of Veterans Affairs Central Office, Geriatrics and Extended Care. They receive additional support from the Houston VA Health Services Research and Development Center for Innovations in Quality, Effectiveness, and Safety (CIN13-413).

Competing interests None declared.

Patient consent for publication Not required.

Provenance and peer review Commissioned; internally peer reviewed.

ORCID iD

Aanand D Naik http://orcid.org/0000-0001-6936-7984

\section{REFERENCES}

1 Institute of Medicine, Plsek P. Crossing the quality chasm: a new health system for the 21st century. Washington, DC: National Academies Press, 2001.

2 Frampton SB. Healthcare and the patient experience: harmonizing care and environment. HERD 2012;5:3-6.

3 Berwick DM. What 'Patient-Centered'Should Mean: Confessions Of An Extremist: A seasoned clinician and expert fears the loss of his humanity if he should become a patient. Health Aff 2009;28:w555-65.

4 Manary MP, Boulding W, Staelin R, et al. The patient experience and health outcomes. N Engl J Med 2013;368:201-3.

5 Davidson KW, Shaffer J, Ye S, et al. Interventions to improve Hospital patient satisfaction with healthcare providers and systems: a systematic review. BMJ Qual Saf 2017;26:596-606.

6 Naik AD. On the road to patient centeredness. JAMA Intern Med 2013;173:218-9.

7 Lee TH. Physician burnout and patient experience: flip sides of the same coin. NEJM Catalyst 2016;2.

8 Starr P. The social transformation of American medicine: the rise of a sovereign profession and the making of a vast industry. Basic Books, 2008.

9 Boyd C, Smith CD, Masoudi FA, et al. Decision making for older adults with multiple chronic conditions: Executive summary for the American geriatrics Society guiding principles on the care of older adults with multimorbidity. J Am Geriatr Soc 2019;67:665-73.

10 Tinetti ME, Naik AD, Dodson JA. Moving from DiseaseCentered to patient Goals-Directed care for patients with multiple chronic conditions: patient value-based care. JAMA Cardiol 2016;1:9-10.

11 Boyd CM, Darer J, Boult C, et al. Clinical practice guidelines and quality of care for older patients with multiple comorbid diseases: implications for pay for performance. JAMA 2005;294:716-24.

12 Naik AD, Dindo LN, Van Liew JR, et al. Development of a clinically feasible process for identifying individual health priorities. J Am Geriatr Soc 2018;66:1872-9.

13 Tinetti M, Dindo L, Smith CD, et al. Challenges and strategies in patients' health priorities-aligned decision-making for older adults with multiple chronic conditions. PLoS One 2019; 14:e0218249.

14 Tinetti ME, Naik AD, Dindo L, et al. Association of patient Priorities-Aligned decision-making with patient outcomes and ambulatory health care burden among older adults with multiple chronic conditions: a nonrandomized clinical trial. JAMA Intern Med 2019;179:1688. 
15 Freytag J, Dindo L, Catic A, et al. Feasibility of clinicians aligning health care with patient priorities in geriatrics ambulatory care. J Am Geriatr Soc 2020;68:2112-6.

16 Feder SL, Kiwak E, Costello D, et al. Perspectives of patients in identifying their values-based health priorities. J Am Geriatr Soc 2019;67:1379-85.

17 Giovannetti ER, Clair CA, Jennings LA. Standardised approach to measuring goal-based outcomes among older disabled adults: results from a multisite pilot. BMJ Qual Saf 2021;30:157-66.

18 Berntsen GKR, Gammon D, Steinsbekk A, et al. How do we deal with multiple goals for care within an individual patient trajectory? A document content analysis of health service research papers on goals for care. BMJ Open 2015;5:e009403.

19 Heisler M, Vijan S, Anderson RM, et al. When do patients and their physicians agree on diabetes treatment goals and strategies, and what difference does it make? J Gen Intern Med 2003;18:893-902.

20 Brown VA, Bartholomew LK, Naik AD. Management of chronic hypertension in older men: an exploration of patient goal-setting. Patient Educ Couns 2007;69:93-9.

21 Bernacki RE, Block SD, American College of Physicians High Value Care Task Force. Communication about serious illness care goals: a review and synthesis of best practices. JAMA Intern Med 2014;174:1994-2003.

22 Rockwood K, Stadnyk K, Carver D, et al. A clinimetric evaluation of specialized geriatric care for rural dwelling, frail older people. J Am Geriatr Soc 2000;48:1080-5.

23 Rockwood K, Howlett S, Stadnyk K, et al. Responsiveness of goal attainment scaling in a randomized controlled trial of comprehensive geriatric assessment. J Clin Epidemiol 2003;56:736-43.
24 Lavallee DC, Chenok KE, Love RM, et al. Incorporating patient-reported outcomes into health care to engage patients and enhance care. Health Aff 2016;35:575-82.

25 Black N, Varaganum M, Hutchings A. Relationship between patient reported experience (PREMs) and patient reported outcomes (PROMs) in elective surgery. BMJ Qual Saf 2014;23:534-42.

26 Varagunam M, Hutchings A, Black N. Do patient-reported outcomes offer a more sensitive method for comparing the outcomes of consultants than mortality? A multilevel analysis of routine data. BMJ Qual Saf 2015;24:195-202.

27 Black N. Patient reported outcome measures could help transform healthcare. BMJ 2013;346:f167.

28 Welch GW, Jacobson AM, Polonsky WH. The problem areas in diabetes scale. An evaluation of its clinical utility. Diabetes Care 1997;20:760-6.

29 Cella D, Riley W, Stone A, et al. The patient-reported outcomes measurement information system (PROMIS) developed and tested its first wave of adult self-reported health outcome item banks: 2005-2008. J Clin Epidemiol 2010;63:1179-94.

30 Turner-Stokes L. Goal attainment scaling and its relationship with standardized outcome measures: a commentary. J Rehabil Med 2011;43:70-2.

31 Naik AD, Martin LA, Moye J, et al. Health values and treatment goals of older, Multimorbid adults facing lifethreatening illness. J Am Geriatr Soc 2016;64:625-31.

32 Naik AD, McCullough LB. Health intuitions inform patientcentered care. Am J Bioeth 2014;14:1-3.

33 Naik AD, Hundt NE, Vaughan EM, et al. Effect of TelephoneDelivered collaborative goal setting and behavioral activation vs enhanced usual care for depression among adults with uncontrolled diabetes: a randomized clinical trial. JAMA Netw Open 2019;2:e198634. 